\section{Proteção da saúde de trabalhadores rurais: a necessidade de padronização das metodologias de quantificação da exposição dérmica a agrotóxicos}

\author{
Health protection for rural workers: the need to \\ standardize techniques for quantifying dermal \\ exposure to pesticides
}

\section{La protección de la salud de los trabajadores rurales: la necesidad de estandarización de las metodologías de cuantificación de la exposición cutánea a los pesticidas}

\begin{abstract}
Quantification of dermal exposure to pesticides in rural workers, used in risk assessment, can be performed with different techniques such as patches or whole body evaluation. However, the wide variety of methods can jeopardize the process by producing disparate results, depending on the principles in sample collection. A critical review was thus performed on the main techniques for quantifying dermal exposure, calling attention to this issue and the need to establish a single methodology for quantification of dermal exposure in rural workers. Such harmonization of different techniques should help achieve safer and healthier working conditions. Techniques that can provide reliable exposure data are an essential first step towards avoiding harm to workers' health.
\end{abstract}

Pesticides; Pesticide Exposure; Workers
Giuliana da Fontoura Rodrigues Selmi 1 Angelo Zanaga Trapé 1

A quantificação da exposição dérmica a agrotóxicos, utilizada no processo de avaliação do risco à saúde de trabalhadores rurais, pode ser realizada usando-se diferentes metodologias, como patches ou corpo total. A existência de diversos métodos pode ser considerada uma limitação relacionada ao processo, já que podem não produzir resultados similares devido às diferenciações nos princípios envolvidos na coleta das amostras. Dessa maneira, realizou-se uma revisão crítica das principais metodologias utilizadas para a quantificação da exposição dérmica, com o objetivo de ressaltar a importância da discussão sobre o assunto e de evidenciar a necessidade de ações que contribuam para o estabelecimento de uma metodologia única para a quantificação da exposição dérmica de trabalhadores rurais. Entende-se que a harmonização das metodologias contribuirá para o alcance de condições mais seguras e saudáveis de trabalho, principalmente por possibilitar a obtenção de resultados mais confiáveis e, portanto, estabelecer, garantir e aprimorar o processo de prevenção de agravos à saúde do trabalhador.

Agrotóxicos; Exposição a Praguicidas; Trabalhadores 


\section{Introdução}

Diariamente em todo o mundo um grande número de substâncias químicas são produzidas, transportadas e armazenadas, gerando um significante potencial de exposição do homem a estes produtos, tanto pela frequência de uso, como pela diversidade de substâncias existentes. Dentre esses produtos podemos citar os agrotóxicos 1,2.

Os agrotóxicos são substâncias que possuem como principal finalidade a proteção dos produtos agrícolas contra a ação de seres vivos nocivos, como alguns tipos de insetos, por exemplo. Por serem amplamente utilizados na agricultura, apresentam significativo potencial de exposição, principalmente dos trabalhadores rurais que possuem como rotina de trabalho o manuseio desses produtos. Dentro desse contexto, não se pode esquecer o papel do trabalhador agrícola e dos impactos do manuseio de agrotóxicos em sua saúde. Dessa maneira, é uma necessidade gerar condições seguras e adequadas de trabalho, nas quais torna-se imprescindível a adoção de medidas de prevenção e proteção à saúde dos trabalhadores que misturam, carregam e aplicam agrotóxicos 3,4 .

As orientações para a proteção da saúde humana e do meio ambiente são fornecidas com base nas características dos agrotóxicos. Esses cuidados são determinados com base no conhecimento de questões como a toxicidade do ingrediente ativo, a forma de apresentação e de aplicação, assim como de características da cultura alvo e da extensão da área de aplicação 5,6.

Dessa forma verifica-se que diversos fatores contribuem para o estabelecimento do cenário final de exposição do trabalhador ${ }^{4}$, que juntamente com as propriedades toxicológicas do ingrediente ativo podem direcionar para uma melhor avaliação das margens de segurança. Isso é também conhecido como avaliação do risco, processo amplamente utilizado na avaliação de segurança de agrotóxicos 5 .

A avaliação do risco é um processo sistemático, no qual o perigo, a exposição e o risco são identificados e quantificados 5,6,7,8. Os elementos constituintes desse processo incluem: descrição dos efeitos adversos potenciais e avaliação dos resultados de estudos epidemiológicos, clínicos e toxicológicos com animais de experimentação; extrapolação destes resultados para predizer o tipo e estimar a extensão dos efeitos à saúde do homem sob condições específicas da exposição; análise quanto ao número e às características dos indivíduos expostos à diferente intensidade e duração; e por fim, na caracterização do risco, realização da síntese destes elementos, que expressa a existência e magnitude do problema de saúde 5,9. Essa última etapa representa um importante elo de ligação entre os dados científicos obtidos nos diferentes estudos e as decisões governamentais quanto à regulamentação, assim como quanto às decisões de ordem política sobre o gerenciamento e a comunicação do risco 9,10.

A avaliação da exposição, uma das etapas que compõem o processo de avaliação do risco, é uma etapa que visa mensurar a intensidade, a frequência e a duração da exposição aos agrotóxicos, sendo extremamente importante para a determinação das margens de segurança no manuseio e aplicação destes produtos 11,12,13.

A exposição pode ser estimada diretamente, por meio da condução do estudo em campo, ou indiretamente, usando-se os modelos preditivos da exposição 3,4,5,7,8,14,15,16,17,18. Esses são modelos matemáticos que reúnem informações de exposição provenientes de diversos estudos de monitoramento já realizados com trabalhadores que misturam, carregam e aplicam agrotóxicos em condições típicas e reais de campo. Os dados constituintes dos modelos preditivos são provenientes de estudos de monitoramento em campo por dosimetria passiva, nos quais a quantidade de agrotóxico depositada nas roupas, pele e na área de respiração do trabalhador, durante as tarefas de preparo, carregamento e aplicação, foi quantificada. Em resumo, a metodologia chamada de dosimetria passiva consiste na coleta e quantificação de todo resíduo depositado sobre os dosímetros distribuídos pelo corpo do trabalhador e que simulam sua pele ou área de inalação 19,20.

O estudo de monitoramento em campo, além de fornecer ferramentas para a quantificação da exposição a agrotóxicos e, consequentemente possibilitar a avaliação da exposição de trabalhadores, pode também ser utilizado para a verificação da eficácia dos equipamentos de proteção individual recomendados durante a prática agrícola 21 . Esse processo pode ser realizado para a confirmação do equipamento de proteção individual recomendado, ou, ao contrário, pode contradizer a recomendação inicial possibilitando ajustes e adequações para a correta proteção do trabalhador.

A exposição ocupacional aos agrotóxicos ocorre principalmente através das vias inalatória e dérmica. Nos últimos anos tem-se observado uma maior preocupação quanto à importância da exposição através da via dérmica, até porque um significante progresso já foi evidenciado quanto ao desenvolvimento de uma metodologia única para se mensurar a exposição através da via inalatória. Entretanto, quando fazemos referência à definição de uma metodologia para mensuração da exposição dérmica, o cenário 
ainda não é suficientemente estabelecido, considerando a existência de uma ampla variedade de técnicas possíveis de quantificação da exposição por esta via 19. Exemplificando este fato, entre as técnicas de dosimetria passiva disponíveis para quantificação da exposição dérmica pode-se citar algumas, como a utilização de patches, corpo total ou traçadores fluorescentes, corantes visíveis, assim como técnicas de lavagem ou limpeza com água e sabão ou com solvente específico.

A dosimetria passiva é uma metodologia utilizada desde os anos 1950, sendo descrita por Durhan \& Wolf (1962, apud Organisation for Economic Co-operation and Development 22), a qual, agregada a outras publicações em anos seguintes como de Wolf (1976, apud Organisation for Economic Co-operation and Development 22) e Davis (1980, apud Organisation for Economic Co-operation and Development 22), serviram como base para a elaboração do protocolo de avaliação da exposição da Organização Mundial da Saúde (OMS) de 1975 (apud Organisation for Economic Co-operation and Development 22) e de 1982 (apud Organisation for Economic Co-operation and Development 22 ).

Nessa época, as metodologias de dosimetria passiva estavam sendo desenvolvidas e, deste modo, entre as décadas de 1950 e 1970, um grande número de estudos foram conduzidos 14 . Inicialmente utilizava-se somente a técnica dos patches, os quais eram localizados externamente à roupa de trabalho em cerca de seis regiões do corpo e não havia nenhum protocolo que orientasse a condução do estudo. Com o tempo, os patches passaram a ser localizados também sob a vestimenta de trabalho, considerando também a quantidade de produto que alcançaria a pele do trabalhador, e o número de patches utilizados passou para cerca de dez, aumentando com isto a área de coleta de amostra 14 . O primeiro protocolo desenvolvido pela OMS, em 1975, tinha como objetivo principal estipular parâmetros para quantificar a exposição aos inseticidas organofosforados. Em 1980, surgiu a técnica de dosimetria passiva conhecida atualmente como corpo total (whole body), sendo que a revisão do protocolo da OMS, em 1982, permitiu a sua aplicabilidade para as demais classes de agrotóxicos, além de incluir a metodologia do corpo total para avaliação da exposição dérmica, já que os primeiros protocolos citavam somente o método patch como metodologia disponível para esta avaliação. Finalmente, no final dos anos 90, agências internacionais como a Organização para a Cooperação e Desenvolvimento Econômico (OECD) e a Environmental Protection Agency dos Estados Unidos (EPA) criaram protocolos para orientar a condução dos estudos para a quan- tificação da exposição dérmica. Ainda hoje esses são os guias utilizados para a condução desse tipo de estudo $8,14,22$.

É importante observar que esses protocolos internacionais ainda propõem a possibilidade de utilização de duas ou mais metodologias distintas para a avaliação da exposição dérmica 23, além de não haver uma legislação nacional que oriente a realização e condução dos estudos de monitoramento ocupacional no Brasil. Nesse contexto, os diversos métodos existentes podem ser considerados uma das limitações associadas à avaliação da exposição dérmica, já que frequentemente não produzem resultados similares devido às diferenciações nos princípios envolvidos na coleta das amostras 17,19.

Com base no exposto, este trabalho tem como objetivo levantar as limitações envolvidas no processo de quantificação da exposição dérmica e, desta maneira, ressaltar a importância e a necessidade de ações que contribuam para o desenvolvimento de uma metodologia nacional padronizada para quantificação da exposição dérmica de trabalhadores rurais durante a condução dos estudos de monitoramento ocupacional em campo.

\section{Metodologia}

O texto faz referência às principais metodologias disponíveis utilizadas para a condução dos estudos de monitoramento ocupacional em campo, e foi desenvolvido usando-se o método exploratório e de pesquisa bibliográfica. Como base principal para a discussão, foram utilizados os protocolos internacionais específicos que orientam acerca da condução deste tipo de estudo, os da serie 875 da EPA 22,23,24 e o guia no 9 da OECD 22 . Para a complementação das informações, foi também realizada uma ampla busca bibliográfica em literatura nacional e internacional, compreendendo livros, documentos relacionados ao assunto proposto, documentos publicados de agências governamentais, assim como publicações de universidades internacionais e produção científica proveniente de artigos veiculados em revistas indexadas e em banco de dados e sistemas de bibliotecas presenciais ou virtuais, oriundos de fontes seguras e cientificamente reconhecidas.

\section{Metodologias de dosimetria passiva (via dérmica)}

Os protocolos internacionais são fundamentais e possibilitam a utilização de diversas metodo- 
logias de dosimetria passiva para a condução do estudo em campo, como: o método de adesivos (patch), o método que utiliza traçadores fluorescentes e corantes visíveis e o método do corpo total (whole body) 4,14,17,19,24,25,26,27,28,29.

$\mathrm{O}$ método patch refere-se à utilização de pedaços de tecido, geralmente de algodão, com dimensões conhecidas, que são fixados ao indivíduo em regiões preestabelecidas do corpo, tanto na parte externa quanto na parte interna da vestimenta. Geralmente a área coberta pelos patches representa menos de $10 \%$ da área total da superfície do corpo; esta recomendação é encontrada nos guias da EPA e da OECD. Em contrapartida, no protocolo da OMS de 1982 a representação da superfície corpórea pelos patches é de apenas $3 \%$ 4,14,15,19,22,30,31,32,33,34. Os resíduos depositados nos patches são quantificados e o resultado é extrapolado para a área corpórea total. Ao utilizar esse método, assume-se que a deposição do produto ocorre de forma uniforme em todas as regiões do corpo do indivíduo, ocasionando uma análise superestimada ou subestimada da exposição 15,19,22,31,33,35,36.

O método que utiliza traçadores fluorescentes ou corantes visíveis envolve o tratamento da substância teste com um traçador fluorescente e posterior utilização de vídeo imagem para a realização de análise visual e quantitativa, também com o objetivo de identificar as regiões do corpo onde a deposição da substância ocorreu. O traçador fluorescente ou o corante visível pode também ser extraído de dosímetros e analisados, assim como a substância de interesse 15,24,25,31,35,37. Essa técnica permite identificar padrões de exposição ao produto e cenário de exposição específico, sendo muito utilizada no treinamento de trabalhadores, pois proporciona a visualização do produto depositado sobre o corpo dos indivíduos, assim como as regiões mais atingidas, permitindo demonstrar a extensão da exposição, além de facilitar a compreensão dos trabalhadores em relação às práticas agrícolas, contribuindo para a mudança de hábitos e consequente redução da exposição 22,25,31,36.

O método conhecido como corpo total é caracterizado pela utilização de duas camadas de roupa cobrindo todo o corpo do trabalhador (geralmente de algodão, como calças compridas e camisa de manga longa ou macacão inteiriço), atuando como um meio de coleta da substância teste, que se depositaria sobre a pele. A camada de roupa externa representa a vestimenta de trabalho, e a camada de roupa interna representa a pele do indivíduo 4,15,19,21,22,31,32,33,36,38. Qualquer outro equipamento de proteção individual (EPI) recomendado durante o manuseio do produto é utilizado acima da roupa de amostragem, possi- bilitando também uma avaliação da eficiência do EPI 22,33.

A exposição das mãos do trabalhador pode ser avaliada pela utilização de luvas de algodão, por meio de lavagem das mãos com água e sabão ou da limpeza (wipes) com outro solvente específico, removendo os resíduos depositados nesta região do corpo. A exposição das mãos representa uma importante etapa do processo de avaliação da exposição. Essa afirmação é proveniente de diversos estudos que analisaram especificamente a contribuição da exposição das mãos frente à exposição total, verificando que a contribuição pode variar de cerca de 40 a $98 \%$ da exposição total, dependendo de diversas características, como a função do trabalhador, o tipo de aplicação e as propriedades da formulação $15,22,30,31,32,33,34,35,38,39$.

Os resíduos depositados na face e no pescoço do indivíduo são removidos por meio de limpeza destas regiões com gaze embebida em água e sabão ou outro solvente apropriado (wipes) $14,15,19,22,32,35,38$.

A Tabela 1 relaciona as metodologias de dosimetria passiva disponíveis para quantificação da exposição dérmica com as respectivas regiões do corpo.

\section{Cenário atual}

A discussão sobre o assunto no Brasil é recente. Somente em 2002 foi publicado o Decreto no 4.074 40, que regulamenta a Lei no 7.802/198941, e estabelece um prazo para a elaboração de critérios para a implementação da avaliação do risco de agrotóxicos para fins regulatórios. Desde então, não mais observou-se o desenvolvimento de ações concretas no setor, principalmente quanto à etapa de avaliação da exposição, especialmente no que se refere ao estabelecimento dos critérios nacionais para a condução deste tipo de estudo. Desse modo, até o momento não há uma metodologia nacional estabelecida que oriente a realização e condução dos estudos de monitoramento ocupacional em campo no Brasil, o que ressalta, portanto, a importância dos protocolos internacionais existentes sobre o assunto, os quais são a base para a condução deste tipo de estudo.

Já internacionalmente a importância da coleta adequada de dados para a avaliação da exposição de trabalhadores vem sendo discutida e implementada. Para tanto, foi criada uma força-tarefa, a Agricultural Handler Exposure Task Force (AHETF), que é composta por mais de 20 empresas do setor agrícola mundial. Essa força-tarefa tem gerado novos dados de exposição dérmica e 
Tabela 1

Regiões do corpo avaliadas por dosimetria passiva.

\begin{tabular}{|c|c|c|}
\hline Via de exposição & Região do corpo & Principais técnicas de dosimetria passiva \\
\hline \multirow[t]{8}{*}{ Dérmica } & Tronco, membros superiores e inferiores & Patches \\
\hline & & Corpo total \\
\hline & & Traçadores fluorescentes/corantes visíveis \\
\hline & Mãos & Lavagem com água e sabão \\
\hline & & Lavagem com solvente específico \\
\hline & & Luvas impermeáveis \\
\hline & & Luvas de algodão \\
\hline & Face e pescoço & $\begin{array}{c}\text { Limpeza com gaze embebida em água e sabão ou } \\
\text { solvente apropriado (wipes) }\end{array}$ \\
\hline
\end{tabular}

inalatória de trabalhadores durante o manuseio e aplicação de agrotóxicos. Entre os objetivos da AHETF pode-se citar a necessidade de identificação de problemas nos dados de estudos de exposição conduzidos no passado, a condução de novos estudos abrangendo também cenários de uso mais atuais, e a criação de um modelo preditivo, o AHED (Agricultural Handlers Exposure Database), um banco de dados mais recente 42 .

A condução de novos estudos pela AHETF também tem como objetivo a obtenção de dados mais significativamente relevantes para a estimativa da exposição, já que novos estudos irão melhor refletir as práticas agrícolas em uso atualmente, cobrindo assim as lacunas existentes nos demais modelos preditivos de exposição. Segundo a AHETF, o desenvolvimento de metodologias mais confiáveis de estimativa da exposição em campo é ponto fundamental para que esses objetivos sejam alcançados 43 .

\section{Discussão e considerações finais}

Todos esses métodos têm vantagens e limitações. O método patch exige que seja realizada a extrapolação dos dados de uma pequena área do corpo para a área total, assumindo-se com isto uma deposição uniforme dos resíduos. Essa pode ser considerada a principal limitação associada a essa técnica, que pode acarretar uma superestimativa ou uma subestimativa da exposição.

Essa limitação é suprimida com a utilização da técnica do corpo total, que fornece uma amostragem do corpo inteiro do trabalhador. Essa técnica permite a coleta e quantificação de todo o resíduo depositado sobre o indivíduo. Não há portanto a necessidade de se assumir uma deposição uniforme do resíduo e, consequentemente, não precisam ser extrapolados. Ao mesmo tempo, a utilização de duas camadas de vestimenta oferece certo desconforto ao trabalhador, principalmente em regiões, épocas do ano e horários que apresentam temperaturas mais elevadas, além da análise dos resíduos ser mais trabalhosa e, muitas vezes, mais onerosa.

Quanto aos traçadores fluorescentes e corantes visíveis, há a possibilidade de se realizar tanto uma análise visual quanto quantitativa, sendo muito eficaz quando utilizado para o treinamento dos trabalhadores. Entretanto, assume-se equivalente deposição entre o traçador/corante e o agrotóxico.

No caso da avaliação da região das mãos, a utilização de luvas absorventes pode resultar numa significante superestimativa da exposição dérmica total, pois sua capacidade de retenção é maior que a da pele. Luvas também podem conter outras substâncias, que se extraídas juntamente com o agrotóxico podem tornar-se um importante interferente quando se tem baixos níveis de contaminação. Em contrapartida, a utilização de luvas absorventes apresenta grande praticidade na coleta da amostra. A limpeza (wipes) ou lavagem das mãos é considerada, por alguns, como modificador da função de barreira da pele, podendo intensificar a absorção do agrotóxico. Ainda, a limpeza das mãos com swab não tem sido muito satisfatória devido à dificuldade de se limpar adequadamente entre os dedos e nas unhas. Outras variáveis que também podem interferir na eficiência da amostragem pelos métodos de lavagem das mãos são: o número de lavagens consecutivas realizadas, o vigor e o tempo de lavagem.

Portanto, verifica-se que existem distintos métodos utilizados e fica claro observar que a amplitude de possibilidades acaba por não per- 
mitir uma padronização adequada na condução deste tipo de estudo, podendo ser este fator considerado como uma das limitações associadas à avaliação da exposição dérmica 3,8,17,19.

Sabe-se também que, dentro do processo de avaliação do risco, os estudos de monitoramento ocupacional em campo podem refletir as reais condições de exposição e fornecer subsídios para a melhor avaliação da segurança e proteção da saúde do trabalhador ${ }^{3}$. Por esse motivo, justificase a importância de se discutir este assunto, em função da ampla utilização de agrotóxicos e do grande número de agricultores que atuam nesta função.

Outro ponto relevante a ser observado referese à escassez de dados provenientes de estudos de monitoramento ocupacional em campo para avaliação da exposição, principalmente em âmbito nacional. Poucos trabalhos foram conduzidos no Brasil, assim, a avaliação da exposição apresentada para o setor de regulação nacional é a realizada por meio dos modelos preditivos internacionais que contêm resultados provenientes dos estudos de campo conduzidos em países, na maioria das vezes, com clima, cultura, condições e cenários de aplicação diferentes dos comumente utilizados no Brasil.

Ao mesmo tempo, tem sido dada pouca ênfase no desenvolvimento de um modelo básico de avaliação da exposição dérmica, e esta lacuna é um dos principais motivos da falta de uma metodologia bem definida de amostragem desta exposição. Todos os métodos descritos podem ser utilizados e possuem vantagens e limitações, cabe ao pesquisador avaliar qual deles, dentro das características do estudo, atende melhor às necessidades 13,44 .

Portanto, observa-se que o processo de avaliação da exposição é fator fundamental para a segurança, saúde e bem-estar da população em geral, principalmente dos trabalhadores agrícolas que manipulam e aplicam agrotóxicos. Dessa forma, qualquer empenho no sentido do desenvolvimento de metodologias harmonizadas e cientificamente embasadas contribui para o alcance de condições mais seguras e saudáveis de trabalho, principalmente por possibilitar a obtenção de resultados mais confiáveis.

A partir do momento em que um estudo é adequadamente delineado, dados de qualidade são gerados, medidas eficientes de proteção são criadas e, por conseguinte, a saúde da população, neste caso especificamente do trabalhador rural, é assegurada. Consegue-se, portanto, estabelecer, garantir e aprimorar o processo de prevenção de agravos à saúde do trabalhador.

Em resumo, a disponibilidade de diversos métodos de avaliação da exposição se torna um problema no que diz respeito à obtenção de resultados comparáveis, devido principalmente às inúmeras diferenças nos princípios envolvidos na coleta das amostras. A falta de uma metodologia bem definida de amostragem da exposição dérmica faz com que a escolha seja baseada nas características do estudo, assim como nas vantagens e limitações dos métodos, cabendo ao pesquisador a difícil decisão quanto ao método a ser utilizado. 


\section{Resumen}

La cuantificación de la exposición cutánea a los pesticidas, utilizada en el proceso de evaluación del riesgo para la salud de los trabajadores rurales, se puede realizar a través de distintos métodos como parches o totalidad del cuerpo. La existencia de varios métodos puede ser considerada como una limitación relacionada con el proceso, ya que puede producir resultados distintos, debido a las diferencias en los principios implicados en la recogida de las muestras. Por lo tanto, se realizó una revisión crítica de las principales metodologías utilizadas para la cuantificación de la exposición cutánea, con la finalidad de destacar la importancia de la discusión sobre el tema y poner en evidencia la necesidad de acciones que contribuyan a la creación de una metodología única para la cuantificación de la exposición cutánea de los trabajadores rurales. Se entiende que la armonización de las metodologías contribuirá a la consecución de condiciones de trabajo más seguras y saludables, porque permite obtener resultados más fiables y, por lo tanto, asegurar y mejorar el proceso de prevención de daños a la salud de los trabajadores.

Plaguicidas; Exposición a Plaguicidas; Trabajadores

\section{Colaboradores}

G. F. R. Selmi contribuiu com a concepção e planejamento do projeto, realizou o levantamento bibliográfico, a análise dos dados e redigiu o artigo. A. Z. Trapé contribuiu com a concepção e planejamento do projeto, orientou o estudo, além de contribuir com a revisão crítica e redação final do artigo.

\section{Agradecimentos}

À Universidade Estadual de Campinas.

\section{Referências}

1. Della Rosa HV, Siqueira MEPB, Colacioppo S. Monitoramento ambiental e biológico. In: Oga S, organizador. Fundamentos de toxicologia. 3a Ed. São Paulo: Editora Atheneu; 2008. p. 241-60.

2. International Programme on Chemical Safety. Environmental health criteria 210: principles for the assessment of risks to human health from exposure to chemicals. http://www.inchem.org/docu ments/ehc/ehc/ehc210.htm (acessado em 26/Jul/ 2012).

3. Geer LA, Dellarco MJ, Leighton TJ, Zendzian RP, Roberts JD, Buckley TJ. Comparative analysis of passive dosimetry and biomonitoring for assessing chlorpyrifos exposure in pesticide workers. Ann Occup Hyg 2004; 48:683-95.
4. Frenich AG, Aguilera PA, Gonzalez FE, Cano MLC, Galera MM, Vidal JLM, et al. Dermal exposure to pesticides in greenhouses workers: discrimination and selection of variables for the design of monitoring programs. Environ Monit Assess 2002; 80:51-63.

5. Corrêa CL, Alonzo HGA, Trevisan RMS. Avaliação do risco. In: Oga S, organizador. Fundamentos de toxicologia. 3ạ Ed. São Paulo: Editora Atheneu; 2008. p. 71-9.

6. Trevisan RMS, Zambrone FAD. Regulamentação do registro de agrotóxico: abordagem da avaliação da exposição e do risco toxicológico ocupacional. São Paulo: ILSI Brasil; 2002. 
7. Hamey PY. An example to illustrate the potential use of probabilistic modelling to estimate operator exposure to pesticides. Ann Occup Hyg 2001; 45:S55-64.

8. Krieger RI, Bernard CE, Dinoff TM, Fell L, Osimitz TG, Ross JH, et al. Biomonitoring and whole body cotton dosimetry to estimate potential human dermal exposure to semivolatile chemicals. J Expo Anal Environ Epidemiol 2000; 10:50-7.

9. Solomon KR, Stephenson GR, Corrêa CL, Zambrone FAD. Agrotóxicos e o meio ambiente. São Paulo: ILSI Brasil; 2010.

10. Faustman EM, Omenn G. Risk assessment. In: Klaassen CD, editor. Casarett \& Doull's toxicology: the basic science of poisons. $6^{\text {th }}$ Ed. New York: McGraw-Hill Professional; 2001. p. 67-81.

11. World Health Organization. Generic risk assessment model for indoor and outdoor space spraying of insecticides. http://whqlibdoc.who.int/pub lications/2010/9789241599542_eng.pdf (acessado em $07 / J u l / 2012$ ).

12. World Health Organization. Harmonization project document no. 3: principles of characterizing and applying human exposure models. http:// www.inchem.org/documents/harmproj/harm proj/harmproj3.pdf (acessado em 07/Jul/2012).

13. Sheldon LS. Exposure framework. In: Krieger R, editor. Handbook of pesticide toxicology. 3rd Ed. London: Academic Press; 2010. p. 971-6.

14. Ross J, Chester G, Driver J, Lunchick C, Holden L, Rosenheck L, et al. Comparative evaluation of absorbed dose estimates derived from passive dosimetry measurements to those derived from biological monitoring: validation of exposure monitoring methodologies. J Expo Sci Environ Epidemiol 2008; 18:211-30.

15. Fenske RA, Day Jr. EW. Assessment of exposure for pesticide handlers in agricultural, residential and institutional environments. In: Franklin CA, Worgan JP, editors. Occupational and residential exposure assessment for pesticides. Chichester: John Wiley \& Sons; 2005. p. 33-43.

16. Marquart J, Brouwer DH, Gijsbers JHJ, Links IHM, Warren N, van Hemmen JJ. Determinants of dermal exposure relevant for exposure modelling in regulatory risk assessment. Ann Occup Hyg 2003; 47:599-607.

17. Schneider T, Cherrie JW, Vermeulen R, Kromhout H. Dermal exposure assessment. Ann Occup Hyg 2000; 44:493-9.

18. U.S. Environmental Protection Agency. PHED surrogate exposure guide. Washington DC: Office of Pesticide Programs, U.S. Environmental Protection Agency; 1998.

19. Soutar A, Semple S, Aitken RJ, Robertson A. Use of patches and whole body sampling for the assessment of dermal exposure. Ann Occup Hyg 2000; 44:511-8.

20. Reinert JC, Nielsen AP, Lunchick C, Hernandez O, Mazzeta DM. The United States Environmental Protection Agency's guidelines for applicator exposure monitoring. Toxicol Lett 1986; 33:183-91.

21. Machera K, Tsakirakis A, Charistou A, Anastasiadou P, Glass CR. Dermal exposure of pesticide applicators as a measure of coverall performance under field conditions. Ann Occup Hyg 2009; 53:573-84.
22. Organisation for Economic Co-operation and Development. Guidance document for the conduct of studies of occupational exposure to pesticides during agricultural application. Paris: Organisation for Economic Co-operation and Development; 1997. (Series on Testing and Assessment, 9).

23. Blanco LE, Aragon A, Lundberg I, Wesseling C, Nise G. The determinants of dermal exposure ranking method (DERM): a pesticide exposure assessment approach for developing countries. Ann Occup Hyg 2008; 52:535-44.

24. Cherrie JW, Brouwer DH, Roff M, Vermeulen R, Kromhout $\mathrm{H}$. Use of qualitative and quantitative fluorescence techniques to assess dermal exposure. Ann Occup Hyg 2000; 44:519-22.

25. Fenske RA. Dermal exposure: a decade of real progress. Ann Occup Hyg 2000; 44:489-91.

26. U.S. Environmental Protection Agency. Dermal exposure assessment: principles and applications. http://cfpub.epa.gov/ncea/cfm/recordisplay.cfm? deid=12188\#Download (acessado em 01/Ago/ 2012).

27. U.S. Environmental Protection Agency. Background for application exposure monitoring test guidelines. EPA 712-C-96-261. Occupational and residential exposure test guidelines. http://www. epa.gov/ocspp/pubs/frs/publications/Test Guidelines/series875.htm (acessado em 27/Fev/ 2012).

28. U.S. Environmental Protection Agency. Dermal exposure. EPA 712-C-96-269. Occupational and residential exposure test guidelines. http://www. epa.gov/ocspp/pubs/frs/publications/Test_Guide lines/series875.htm (acessado em 27/Fev/2012).

29. U.S. Environmental Protection Agency. Dermal exposure - outdoor. EPA 712-C-96-262. Occupational and residential exposure test guidelines. http:// www.epa.gov/ocspp/pubs/frs/publications/Test Guidelines/series875.htm (acessado em 27/Fev/ 2012).

30. Hines CJ, Deddens JA, Coble J, Kamel F, Alavanja MCR. Determinants of captan air and dermal exposures among orchard pesticide applicators in the agricultural health study. Ann Occup Hyg 2011; 55:620-33.

31. Chester G. Worker exposure: methods and techniques. In: Krieger R, editor. Handbook of pesticide toxicology. 3rd Ed. London: Academic Press; 2010. p. 1127-38.

32. Rajan-Sithamparanadarajah R, Roff M, Delgado P, Eriksson K, Fransman W, Gijsbers J, et al. Patterns of dermal exposure to hazardous substances in European Union workplaces. Ann Occup Hyg 2004; 48:285-97.

33. Machera K, Goumenou M, Kapetanakis E, Kalamarakis A, Glass CR. Determination of potential dermal and inhalation operator exposure to malathion in greenhouses with the whole body dosimetry method. Ann Occup Hyg 2003; 47:61-70.

34. Honeycutt RC. NACA overview on assessment of mixer-loader-applicator exposure to pesticides. Toxicol Lett 1986; 33:175-82.

35. van Hemmen JJ, Brouwer DH. Assessment of dermal exposure to chemicals. Sci Total Environ 1995; 168:131-41. 
36. Whitmyre GK, Ross JH, Lunchick C. Occupationa exposure data bases/models for pesticides. In: Krieger R, editor. Handbook of pesticide toxicology: principles. 2nd Ed. London: Academic Press; 2001. p. 493-506.

37. University of Washington. Fluorescent tracer manual: an educational tool for pesticide safety educators. http://depts.washington.edu/pnash/files/ FT/FT_Manual.pdf (acessado em 01/Ago/2012).

38. Cattani M, Cena K, Edwards J, Pisaniello D. Potential dermal and inhalation exposure to chlorpyrifos in Australian pesticide workers. Ann Occup Hyg 2001; 45:299-308.

39. Youngren SH, Youngren MA, Barra L. Challenges of probabilistic assessment of operator and residential non-dietary exposure. Ann Occup Hyg 2001; 45 Suppl 1:S49-54.

40. Brasil. Decreto no 4.074, de 4 de janeiro de 2002. Regulamenta a Lei no 7.802 , de 11 de julho de 1989 , que dispõe sobre a pesquisa, a experimentação, a produção, a embalagem e rotulagem, o transporte, o armazenamento, a comercialização, a propaganda comercial, a utilização, a importação, a exportação, o destino final dos resíduos e embalagens, o registro, a classificação, o controle, a inspeção e a fiscalização de agrotóxicos, seus componentes e afins, e dá outras providências. Diário Oficial União 2002; 8 jan.
41. Brasil. Lei no 7.802, de 11 de julho de 1989. Dispõe sobre agrotóxicos, componentes e afins e dá outras providências. Diário Oficial União 1989; 11 jul.

42. Lunchick C, Evans J, Iyengar S, Selman F, Wicke H. Operator and field worker occupational exposure databases and modeling. In: Krieger R, editor. Handbook of pesticide toxicology. 3rd Ed. London: Academic Press; 2010. p. 1139-55.

43. European Food Safety Authority. Project to assess current approaches and knowledge with a view to development a guidance document for pesticide exposure assessment for workers, operators, bystanders and residents. http://www.efsa.europa. eu/en/publications.htm (acessado em 01/Ago/ 2012).

44. Schneider T, Vermeulen R, Brouwer DH, Cherrie JW, Kromhout H, Fogh CL. Conceptual model for assessment of dermal exposure. Occup Environ Med 1999; 56:765-73.

Recebido em 22/Nov/2012

Versão final reapresentada em 12/Set/2013

Aprovado em 04/Nov/2013 